\title{
Untangling LUTS, BPH and renal function
}

Lower urinary tract symptoms (LUTS), which include storage, voiding and postmicturition symptoms, occur in a considerable proportion of men over the age of 40 years. These findings are often attributed to bladder outlet obstruction secondary to benign prostatic hyperplasia $(\mathrm{BPH})$; however, other conditions are also thought to cause LUTS, such as detrusor overactivity (which is predominantly associated with storage symptoms). In short, the etiology and underlying mechanisms of LUTS are unclear, and the optimal evaluation and management of patients with these symptoms remain elusive. Two large studies provide interesting food for thought regarding these issues.

\section{4 ...the etiology and underlying mechanisms of LUTS are unclear... 77}

The first study, performed by Kaplan and colleagues, examined the safety and efficacy of the antimuscarinic drug fesoterodine as an add-on therapy in men with persistent storage LUTS despite treatment with $\alpha$-blockers. The 943 participants, who were experiencing $\geq 8$ micturitions and $\geq 3$ urgency episodes per day and who had received $\geq 6$ weeks of stable $\alpha$-blocker therapy, were randomly assigned to receive either add-on fesoterodine $4 \mathrm{mg}$ daily $(n=471)$ or placebo $(n=472)$ for 12 weeks. A flexible dosing regime allowed the patients to increase the fesoterodine dose to $8 \mathrm{mg}$ daily at week 4 (an option taken up by just over half of the patients receiving active treatment), and a further option to reduce the dose back down to $4 \mathrm{mg}$ at week 8 .

Fesoterodine was well tolerated, with dry mouth and constipation reported as the most frequent adverse events. The number of urgency episodes per day-the primary end point-did not differ between the fesoterodine and placebo groups at 12 weeks, whereas improvements in symptom bother and the frequency of micturitions were greater in the active treatment group. These findings indicate that, in patients with storage LUTS suggestive of overactive bladder, add-on fesoterodine therapy does not improve urgency.

In the second study, Kwon et al. considered the possible relationship between LUTS and renal function. Early renal dysfunction has been associated with LUTS independently of the presence of bladder outlet obstruction; therefore, the authors tested whether glomerular filtration rate (GFR; a reliable and established marker of renal function) was associated with LUTS after adjustment for age and prostate volume.
The study included data obtained from a cross-sectional survey of 3,713 men aged $\geq 40$ years. The International Prostate Symptom Score (IPSS) was used to assess LUTS severity as mild, moderate or severe. On multivariate regression analysis (adjusted for age), increased severity of LUTS (particularly voiding symptoms) was significantly associated with decreased GFR in men aged $\geq 55$ years. In a stratified analysis by age and prostate volume, GFR was independently and negatively correlated to total IPSS in men aged $\geq 55$ years with small prostates $\left(<30 \mathrm{~cm}^{3}\right)$, and to voiding IPSS in men of this age group with both small and large prostates.

The underlying mechanism that explains this relationship between GFR and LUTS severity is not immediately obvious, but the authors propose that patients with moderate or severe LUTS-including those with normalsized prostates-should have their renal function monitored.

\section{Nick Warde}

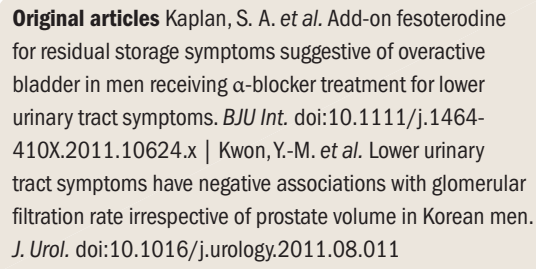

